\title{
IN-BETWEEN THEOREMS IN UNIFORM SPACES
}

BY

\author{
D. PREISS AND J. VILIMOVSKÝ 1
}

\begin{abstract}
Necessary and sufficient conditions for the existence of a uniformly continuous function in-between given functions $f>g$ on a uniform space are studied. It appears that the investigation of this problem is closely related to some combinatorial properties of covers and leads to the concept of perfect refinability, the latter being used, e.g., to obtain an intrinsic description of uniform real extensors. Several interesting classes of uniform spaces are characterized by special types of in-between theorems. As examples of applications we show that the usual in-between theorems in topology and their generalizations, as well as some important methods of construction of derivatives of real functions, follow easily from the general results.
\end{abstract}

0. Introduction. The long history of extension and in-between theorems led to the nice results of $M$. Katětov and $E$. Michael for topological spaces. It appears that in some applications of continuous structures the topological point of view is too restrictive. This, as well as the natural development of in-between problems, led us to formulate and prove the uniform version of these theorems. Although our approach involves quite different ideas, the topological results become easy corollaries. To some extent our ideas are of the obvious sort, but at least one "unstable" property, perfect refinability, ("unstable" here means with respect to usual uniform operations with covers) comes in, in a rather essential way. The results show that the strange definition of perfect refinability is a quite good approximation to a key idea in this subject. Besides the general theory contained in the first two paragraphs we show some interesting applications to topology and the theory of real functions.

Throughout this paper we will refer to [7] for basic definitions and results pertaining to uniform spaces. All uniform spaces are supposed to be Hausdorff. We shall denote by $R$ the real line with its usual metrizable uniformity and usual order, $\bar{R}$ will denote the space of extended reals, i.e. the uniform sum $R \vee\{-\infty\} \vee$ $\{+\infty\}$ with the usual order $-\infty<r<+\infty$ for all $r \in R$. In the text we often simply write function instead of $R$-valued function. For a positive real number $\delta$ we shall denote by $\mathcal{T}(\delta)$ the uniform cover $\{\langle t-(\delta / 2), t+(\delta / 2)\rangle ; t \in R\}$ of $R$.

Received by the editors October 18, 1978 and, in revised form, January 9, 1979.

AMS (MOS) subject classifications (1970). Primary 54E15, 54C30; Secondary 26A24.

Key words and phrases. Uniform space, mixed preimage, far sets with respect to a cover, perfectly refinable cover, Ext-uniformity, inversion-closed space.

${ }^{1}$ We would like to express our thanks to the referee, since we think his remarks have led to considerable improvement of the paper. 
The uniformity of $R$ has a basis consisting of linear covers (each point belongs to at most two of its members) composed of intervals of equal length. Such covers will be called basic.

For any set $X$, if $\mathcal{V}$ is a cover of $X$, we shall use the following usual notation: For $x \in X$ we denote $\operatorname{St}(x, \mathcal{V})=\cup\{V \in \mathcal{V} ; x \in V\}, \operatorname{St} \mathcal{V}=\{\operatorname{St}(x, \mathcal{V}) ; x \in$ $X$ ). If $\mathscr{W}$ is another cover of $X$ we use the symbol $\mathscr{V} \prec \mathcal{V}$ (resp. $\mathscr{W} \prec \mathfrak{V}$ ) for QS refines $\mathcal{V}$ (resp. St $\mathcal{W}$ refines $\mathcal{V}$ ). $\omega$ will denote either the set of all finite ordinal numbers or a uniformly discrete space on the set $\omega$. For each $n \in \omega, n \geqslant 1$, we may define the cover $\operatorname{St}^{n} \mathcal{V}$ for any cover $\mathcal{V}$ of $X$ as $\left\{\operatorname{St}^{n}(x, \mathcal{V}) ; x \in X\right\}$, where $\operatorname{St}^{n}(x, \mathcal{V})=\left\{y \in X\right.$ there exist $V_{1}, \ldots, V_{n} \in \mathcal{V}$ with $x \in V_{1}, y \in V_{n}$, and $V_{i-1}$ $\cap V_{i} \neq \varnothing$ for all $\left.i=2, \ldots, n\right\}$. For convenience we put $\mathrm{St}^{0} \mathcal{V}=\mathcal{V}$. Two subsets $A, B$ of $X$ will be called $\mathcal{V}$-far for some cover $\mathfrak{V}$ of $X$, if no member of $\mathcal{V}$ intersects both $A$ and $B$.

For each uniform space $X$ we shall use the symbol $\operatorname{coz} X$ for the set of all cozero (with respect to uniformly continuous functions) sets in $X$. Two subsets $A, B$ of $X$ will be called proximally far, if there is a finite uniform cover $\mathcal{P}$ of $X$ such that they are $\mathcal{P}$-far; they will be called $\operatorname{coz} X$-proximally far, if there is a finite cozero cover $\mathscr{P}$ of $X$ such that $A, B$ are $\mathscr{P}$-far. Finally we recall that the cover $\mathscr{V}$ of $X$ is called: point-finite, if each $x \in X$ belongs to finitely many $V \in \mathcal{V}$ only, finite-dimensional, if there is a natural number $n$ such that each $x \in X$ belongs to at most $n$ members of $\mathfrak{V}$, star-finite, if each $V \in \mathcal{V}$ intersects only finitely many members of $\mathfrak{V}$, or star-bounded (Euclidean), if each $V \in \mathcal{V}$ intersects at most $n$ members of $\mathcal{V}$ for some (fixed) natural number $n$.

1. In-between theorems for general uniform spaces. We begin with the definition of the following concept, which seems to be of basic importance.

1.1. Definition. If $f, g$ are $\bar{R}$-valued functions on a set $X,\langle r, s\rangle$ any closed interval in $\bar{R}$, we define the mixed preimage $(f, g)^{-1}(\langle r, s\rangle)$ as $\{x \in X \mid f(x) \geqslant r$, $g(x) \leqslant s\}$. Similarly we may define mixed preimages of other types of intervals in $\bar{R}$.

1.2. Proposition. Suppose $f \geqslant g$ are $\bar{R}$-valued functions on a set $X$ such that $f>-\infty, g<+\infty$. Then

(i) for every $\delta>0$ and every $r, s \in R$ with $s-r>\delta$, the sets $\{x \in X \mid f(x)<r\}$, $\{x \in X \mid g(x)>s\}$ are $(f, g)^{-1}(\sigma(\delta / 2))$-far and the latter cover refines the covers $\{\{x \in X \mid f(x)>r\} ; r \in R\},\{\{x \in X \mid g(x)<r\} ; r \in R\}$.

(ii) If $\delta>0$ and $\mathcal{V}$ is a cover of $X$ such that the sets $\{x \in X \mid f(x)<r\}$, $\{x \in X \mid g(x)>s\}$ and $\mathfrak{V}$-far for all $r, s \in R$ with $s-r>\delta$, then the cover $\mathcal{V} \wedge\{\{x \in X \mid f(x)>r\} ; \quad r \in R\} \wedge\{\{x \in X \mid g(x)<r\} ; \quad r \in R\}$ refines $(f, g)^{-1}(\mathcal{T}(\delta / 2))$.

(iii) If $f, g$ are finite, $\delta>0$ and $\mathcal{V}$ is a cover of $X$ such that the sets $\{x \in X \mid f(x)$ $<r\},\{x \in X \mid g(x)>s\}$ are $\mathcal{V}$-far for all $r, s \in R$ with $s-r>\delta$, then $\mathcal{V}$ refines the cover $(f, g)^{-1}(T(\delta / 2))$.

Proof. (i) Suppose that (i) does not hold. Then we can find $\delta>0, r, s \in R$ with $s-r>\delta, x, y \in X$ and $t \in R$ such that $f(x)<r, g(y)>s$ and both $x, y$ belong to 
the set $(f, g)^{-1}(\langle t-(\delta / 2), t+(\delta / 2)\rangle)$. This means that $f(x) \geqslant t-(\delta / 2), g(y)<$ $t+(\delta / 2)$, i.e. $g(y)-f(x) \leqslant \delta$. But the above inequalities give $g(y)-f(x)>s-r$ $>\delta$, which is a contradiction. The rest of (i) is evident.

(iii) Take $V \in \mathcal{V}$ nonempty and choose $r_{0}, s_{0} \in R$ such that both $\{x \in X \mid f(x)$ $\left.<r_{0}\right\} \cap V$ and $\left\{x \in X \mid g(x)>s_{0}\right\} \cap V$ are nonempty. This means that the set of all $r \in R$ such that $V \cap\{x \in X \mid f(x)<r\}=\varnothing$ is nonempty, because it contains at least $s_{0}-2 \delta$. Let $r_{1}$ be its supremum. Similarly we put $s_{1}=\inf \{s \in R ;\{x \in$ $X \mid g(x)>s\} \cap V=\varnothing\}$. Then we have $r_{1}+\delta \geqslant s_{1} \geqslant r_{1}, V \cap\left\{x \in X \mid f(x)<r_{1}\right\}$ $=\varnothing, V \cap\left\{x \in X \mid g(x)>s_{1}\right\}=\varnothing$; hence $V$ is contained in the mixed preimage $(f, g)^{-1}\left(\left\langle\left(r_{1}+s_{1}\right) / 2-\delta / 2,\left(r_{1}+s_{1}\right) / 2+\delta / 2\right\rangle\right)$.

(ii) The proof of (ii) is a simple modification of that of (iii).

Now we shall prove the basic theorem of this paragraph.

1.3. THEOREM (GENERAL IN-BETWEEN THEOREM). Let $f \geqslant g$ be $\bar{R}$-valued functions on a uniform space $X$. Then the following conditions are equivalent:

(1) There is an $\bar{R}$-valued uniformly continuous function $\varphi$ on $X$ such that $f \geqslant \varphi \geqslant$ $g$.

(2) For each $\delta>0$ there is a uniform cover $\mathscr{V}$ of $X$ such that, if $n \in \omega$ and $r, s \in R$ with $s-r>(n+1) \delta$, then the sets $\{x \in X \mid f(x) \leqslant r\},\{x \in X \mid g(x) \geqslant$ s) are $\mathrm{St}^{n} \mathfrak{V}$-far.

Proof. $(1) \Rightarrow(2)$ is easy, taking for $\mathcal{V}$ the $\varphi$-preimage of the cover $\mathcal{T}(\delta / 2) \cup$ $\{\{-\infty\},\{+\infty\}\}$ of $R$.

$(2) \Rightarrow(1)$. We choose a uniform cover $\mathcal{V}_{0}$ of $X$ such that for each $n \in \omega$ and each $r, s \in R$ with $s-r>n+1$, the sets $\{x \in X \mid f(x) \leqslant r\},\{x \in X \mid g(x) \geqslant s\}$ are $\operatorname{St}^{n} \mathcal{V}_{0}$-far. Now we define $\mathcal{V}_{m}=\operatorname{St}^{2^{m}} \mathcal{V}_{0}$ for positive integers $m$ and using induction we also define uniform covers $\mathcal{V}_{-m}$ for all $m \in \omega$, such that

(a) $\mathcal{V}_{-m-1} \prec * \mathcal{V}_{-m}$,

(b) for every $r, s \in R$ with $s-r>2^{-m}$, the sets $\{x \in X \mid f(x) \leqslant r\},\{x \in$ $X \mid g(x) \geqslant s\}$ are $\mathcal{V}_{-m}$-far.

Applying the Alexandrov-Urysohn metrization lemma we obtain a uniformly continuous pseudometric $\rho$ on $X$ such that

$$
\mathscr{V}_{p} \prec \mathcal{T}\left(\rho, 2^{p}\right) \prec \mathscr{V}_{p+1}
$$

for all integers $p$. ( $(\mathcal{T}(\rho, r)$ stands for the cover consisting of all $\rho$-balls with diameter r.)

We put $\varphi(x)=\inf \{f(u)+4 \rho(u, x) ; u \in X, \rho(u, x)<+\infty\}$. Clearly $\varphi(x)<$ $f(x)$ for all $x \in X$. To prove that $\varphi \geqslant g$ it suffices to observe that $g(x) \leqslant f(u)+$ $4 \rho(u, x)$ for $u, x \in X$ with $\rho(u, x)<+\infty$. If $g(x) \leqslant f(u)$, there is nothing to prove, if $g(x)-f(u)>2^{p}$, there is no $V \in \mathfrak{V}_{p}$, containing both $x, u$, hence $\rho(u, x) \geqslant$ $2^{p-1}$. Since $\rho(u, x)<+\infty$ we have $g(x)-f(u)<+\infty$. Taking an integer $q$ such that $2^{q} \leqslant g(x)-f(u) \leqslant 2^{q+1}$, we obtain $g(x) \leqslant f(u)+2^{q+1} \leqslant f(u)+4 \rho(u, x)$.

Now we shall prove that $\varphi(x)-\varphi(y) \leqslant 4 \rho(x, y)$ for all $x, y \in X$, where we put $0=+\infty-(+\infty)=-\infty-(-\infty)$.

(a) If either $\rho(x, y)=+\infty$ or $\varphi(y)=+\infty$, the inequality is evident.

(b) Let $\rho(x, y)<+\infty, \varphi(y)$ finite. For each $\varepsilon>0$ choose $u \in X$ such that 
$\rho(u, y)<+\infty$ and $\varphi(y) \leqslant f(u)+4 \rho(u, y) \leqslant \varphi(y)+\varepsilon$. Then

$$
\rho(u, x) \leqslant \rho(x, y)+\rho(y, u)<+\infty
$$

and

$$
\varphi(x)-\varphi(y) \leqslant 4 \rho(u, x)+f(u)-(f(u)+4 \rho(y, u)-\varepsilon) \leqslant 4 \rho(x, y)+\varepsilon,
$$

hence $\varphi(x)-\varphi(y) \leqslant 4 \rho(y, x)$.

(c) If $\rho(x, y)<+\infty, \varphi(y)=-\infty$, for each $c \in R$ choose $u \in X$ such that $\rho(u, y)<+\infty$ and $f(u)+4 \rho(u, y) \leqslant c$. Then

$$
\varphi(x) \leqslant f(u)+4 \rho(u, x) \leqslant f(u)+4 \rho(y, u)+4 \rho(y, x) \leqslant c+4 \rho(y, x),
$$

hence $\varphi(x)-\varphi(y) \leqslant 4 \rho(y, x)$.

The above inequality implies that $\varphi$ is Lipschitz with respect to $\rho$, hence uniformly continuous.

1.4. Corollary (IN-BeTWeEN THeOREM FOR FINITE FUnCtions). Suppose $f>g$ are $R$-valued functions on a uniform space $X$. Then the following conditions are equivalent:

(1) There is a uniformly continuous function $\varphi$ on $X$ with $f \geqslant \varphi \geqslant g$.

(2) For each basic uniform cover $\mathscr{U}$ of $R$ there is a uniform cover $\mathcal{V}$ of $X$ such that $\mathrm{St}^{n} \mathcal{V}$ refines $(f, g)^{-1}\left(\mathrm{St}^{n}\right.$ Q $)$ for each $n \in \omega$.

(3) For each $\delta>0$ there is a uniform cover $\mathfrak{V}$ of $X$ such that for all $n \in \omega$, $r, s \in R$ with $s-r>(n+1) \delta$, the sets $\{x \in X \mid f(x) \leqslant r\},\{x \in X \mid g(x) \geqslant s\}$ are $\mathrm{St}^{n} \mathfrak{T}$-far.

Proof. $(1) \Rightarrow(2)$. Put $\mathcal{V}=\varphi^{-1}(\mathcal{Q}) . \mathcal{V}$ is a linear uniform cover of $X$ and (2) follows immediately from Definition 1.1.

$(2) \Rightarrow(3)$. Take for $\mathscr{U}$ the basic uniform cover of $R$ consisting of intervals of length $\delta$. The corresponding $\mathcal{V}$ fulfills condition (3) immediately from Proposition 1.2 .

(3) $\Rightarrow(1)$ is a direct consequence of Theorem 1.3.

1.5. COROlLARY (IN-BETWEEN THEOREM FOR BOUNDED FUNCTIONS). If $f \geqslant g$ are bounded $R$-valued functions on a uniform space $X$, the following conditions are equivalent:

(1) There exists $\varphi$ uniformly continuous on $X$ such that $f \geqslant \varphi \geqslant g$.

(2) For each $r, s \in R$ with $r<s$, the sets $\{x \in X \mid f(x) \leqslant r\},\{x \in X \mid g(x) \geqslant s\}$ are proximally far.

Proof. It suffices to prove that (2) implies condition (3) from 1.4. Take $\delta>0$. Let $m_{0} \in \omega$ such that $\left\{x \in X \mid f(x) \leqslant-m_{0}(\delta / 2)\right\}=\varnothing,\left\{x \in X \mid g(x)>m_{0}(\delta / 2)\right\}$ $=\varnothing$. For every integer $m$ with $-m_{0} \leqslant m \leqslant m_{0}$ let $\mathcal{V}_{m}$ be a uniform cover of $X$ such that the sets $\{x \in X \mid f(x) \leqslant m(\delta / 2)\},\{x \in X \mid g(x) \geqslant(m+1)(\delta / 2)\}$ are $\mathcal{V}_{m}$-far. Take a uniform cover $\mathcal{V}$ of $X$ such that

$$
\mathrm{St}^{m_{0}} \mathcal{\bigwedge _ { m = - m _ { 0 } } ^ { m _ { 0 } }} \mathcal{V}_{m}
$$


Then (a) If $s-r>(n+1) \delta$ for $0 \leqslant n \leqslant m_{0}$, then the sets $\{x \in X \mid f(x) \leqslant r\}$, $\{x \in X \mid g(x) \geqslant s\}$ are $\mathrm{St}^{n} \mathscr{V}$-far, since they are $\wedge_{m=-m_{0}}^{m_{0}} \mathcal{V}_{m}$-far.

(b) If $s-r>(n+1) \delta, n>m_{0}$, then at least one of the sets $\{x \in X \mid f(x) \leqslant r\}$, $\{x \in X \mid g(x) \geqslant s\}$ is empty, hence they are $\mathrm{St}^{n} \mathfrak{V}$-far as well.

Now applying 1.4 we finish the proof.

1.6. COROllARY (EXTENSION THEOREM). Let $Y$ be a subspace of a uniform space $X$ and let $h$ be a real-valued function on $Y$. Then the following conditions are equivalent:

(1) There exists a uniformly continuous extension of $h$ over $X$,

(2) For each (basic) uniform cover $\mathcal{Q}$ of $R$ there is a uniform cover $\mathfrak{V}$ of $X$ such that $h^{-1}\left(\mathrm{St}^{n}\right.$ Q $)$ is refined by $\mathrm{St}^{n} \mathfrak{V} \uparrow Y$ for each $n \in \omega$.

(3) For each $\delta>0$ there is a uniform cover $\mathfrak{V}$ of $X$ such that the sets $\{x \in Y \mid h(x)$ $\leqslant r\},\{x \in Y \mid h(x) \geqslant s\}$ are $\mathrm{St}^{n} \mathfrak{V}$-far for all $n \in \omega$ and $r, s \in R$ with $s-r>$ $(n+1) \delta$.

Proof. (1) $\Rightarrow(2)$ is obvious. (2) $\Rightarrow(3)$ follows immediately from 1.2 . To prove (3) $\Rightarrow$ (1) we use Theorem 1.3 with $f(x)=g(x)=h(x)$ for $x \in Y, f(x)=+\infty$, $g(x)=-\infty$ otherwise. The function

$$
H(x)=\left\{\begin{array}{l}
\varphi(x), \text { if } \varphi(x) \text { is finite, } \\
0, \text { otherwise, }
\end{array}\right.
$$

is a desired uniformly continuous extension of $h$.

1.7. ThEOREM (STRICT IN-BETWEEN THEOREM). Suppose $f \geqslant g$ are $\bar{R}$-valued functions on a uniform space $X$. Suppose there is a uniformly continuous function $h$ on $X$ such that $f \geqslant h \geqslant g$. Then the following conditions are equivalent:

(1) There exist uniformly continuous functions $\varphi, \psi$ on $X$ such that $f \geqslant \varphi \geqslant \psi \geqslant g$ on $X$ and $f>\varphi>\psi>g$ on the set $A=\{x \in X \mid f(x)>g(x)\}$.

(2) The set $A$ can be covered by countably many cozero (in $X$ ) sets $C_{n}$ such that

$$
\sup _{x \in C_{n}} g(x)<\inf _{x \in C_{n}} f(x) \text { for all } n .
$$

(3) $A$ is a cozero set in $X$ and for every $r, s \in R$ with $r<s$, the sets $\{x \in A \mid f(x)$ $\leqslant r\},\{x \in A \mid g(x) \geqslant s\}$ are $\operatorname{coz} X$-proximally far.

Proof. The implications (1) $\Rightarrow(2),(2) \Leftrightarrow(3)$ are clear.

$(2) \Rightarrow(1)$. Replacing, if necessary, $f$ and $g$ by $\min (f, h+1)$ and $\max (g, h-1)$, we may suppose that $f$ and $g$ are finite. Let $\varphi_{n}$ be uniformly continuous functions on $X$ such that $0 \leqslant \varphi_{n} \leqslant 1$ and $C_{n}=\left\{x \in X \mid \varphi_{n}(x)>0\right\}$. Then

$$
\rho(x, y)=|h(x)-h(y)|+\sum\left(1 / 2^{n}\right)\left|\varphi_{n}(x)-\varphi_{n}(y)\right|
$$

is a uniformly continuous pseudometric on $X$. We put

$$
\begin{aligned}
& \varphi(x)=\inf _{u \in X}(f(u)+2 \rho(u, x)), \\
& \psi(x)=\sup _{u \in X}(g(u)-2 \rho(u, x)) .
\end{aligned}
$$


Then we have

$$
\begin{aligned}
& g(x) \leqslant \psi(x) \leqslant \sup _{u \in X}(h(u)-|h(u)-h(x)|) \leqslant h(x), \\
& f(x) \geqslant \varphi(x) \geqslant \inf _{u \in X}(h(u)+|h(u)-h(x)|) \geqslant h(x) .
\end{aligned}
$$

Similarly, as in the proof of Theorem 1.3, we can prove that both $\varphi, \psi$ are Lipschitz with respect to $\rho$, hence uniformly continuous on $X$.

For $x \in A$ choose $n$ such that $x \in C_{n}$ and put

$$
\varepsilon=\min \left(\left(1 / 2^{n}\right) \varphi_{n}(x), \inf _{u \in C_{n}} f(u)-\sup _{u \in C_{n}} g(u)\right) .
$$

Suppose that $\varphi(x)-\psi(x)<\varepsilon$. Then there are points $u, v \in X$ such that

$$
(f(u)+2 \rho(u, x))-(g(v)-2 \rho(v, x))<\varepsilon,
$$

hence

$$
\begin{aligned}
2(\rho(u, x)+\rho(v, x)) & <\varepsilon+g(v)-f(u) \leqslant \varepsilon+h(v)-h(u) \\
& \leqslant \varepsilon+\rho(u, v) \leqslant \varepsilon+\rho(u, x)+\rho(x, v) .
\end{aligned}
$$

It follows that $\rho(u, x)+\rho(v, x)<\varepsilon$, consequently $u, v \in C_{n}$ and

$$
\varepsilon>(f(u)+2 \rho(u, x))-(g(v)-2 \rho(v, x)) \geqslant f(u)-g(v) \geqslant \varepsilon .
$$

The last contradiction gives that $\varphi>\psi$ on $A$ and the proof is finished.

2. Perfectly refinable covers and in-between theorems for special spaces.

2.1. Definition. Let $X$ be a uniform space, $Y$ its subspace. A uniform cover $\mathscr{U}$ of $Y$ is said to be perfectly refinable in $X$, if for each finite pseudometric $\rho$ on $\mathcal{U}$ there is a uniform cover $\mathcal{V}$ of $X$ such that

$$
\mathrm{St}^{k} \mathscr{V} \uparrow Y \prec\left\{\bigcup_{\rho(U, V)<k} V ; U \in \mathscr{Q}\right\}
$$

for all $k \in \omega$. If $Y=X$, the cover $\mathscr{Q}$ is simply called perfectly refinable.

2.2. Proposition. Let $X$ be a uniform space, $Y$ its subspace, $\mathscr{U}=\left\{U_{\alpha} ; \alpha \in A\right\} a$ countable uniform cover of $Y$. Then the following conditions are equivalent:

(1) $\mathcal{Q}$ is perfectly refinable in $X$.

(2) For each mapping $a: A \rightarrow \omega$ there is a uniform cover $\mathcal{V}$ of $X$ such that

$$
\mathrm{St}^{k} \mathscr{V} \uparrow Y \prec\left\{\bigcup_{|a(\alpha)-n|<k} U_{\alpha} ; n \in \omega\right\}
$$

for all $k \in \omega$.

(3) For each finite separable pseudometric $\rho$ on $A$ there is a uniform cover $\mathcal{V}$ of $X$ such that

$$
\mathrm{St}^{k} \mathscr{V} \uparrow Y \prec\left\{\bigcup_{\rho(\alpha, \beta)<k} U_{\beta} ; \alpha \in A\right\}
$$

for all $k \in \omega$. 
Proof. (1) $\Rightarrow(2)$ is easy, choosing for each $U \in \mathcal{Q}$ some index $\alpha(U)$ from $A$ such that $U=U_{\alpha(U)}$ and then taking the pseudometric $\rho(U, V)=\mid a(\alpha(U))-$ $a(\alpha(V))$.

(3) $\Rightarrow(1)$ is obvious.

$(2) \Rightarrow(3)$. Take a pseudometric space $(A, \rho)$ and choose a countable dense subset $\left\{\gamma_{n} ; n \in \omega\right\}$ of $(A, \rho)$. For each $\alpha \in A$ we put

$$
\begin{aligned}
& n(\alpha)=\min \left\{n \in \omega ; \rho\left(\alpha, \gamma_{n}\right)<1 / 2\right\}, \\
& a(\alpha)=\sum_{i, j<n(\alpha)} 2\left(\left[\rho\left(\gamma_{i}, \gamma_{j}\right)\right]+2\right) .
\end{aligned}
$$

Here $[t]$ denotes the integer part of $t$. If, for some $\alpha, \beta \in A$ we have $|a(\alpha)-n| \leqslant$ $k,|a(\beta)-n| \leqslant k$, then either $n(\alpha)=n(\beta)$, hence $\rho(\alpha, \beta)<1$, or we may suppose that $n(\alpha)<n(\beta)$, hence

$$
\begin{aligned}
2 k & \geqslant a(\alpha)-a(\beta) \geqslant 2\left(\left[\rho\left(\gamma_{n(\beta)}, \gamma_{n(\alpha)}\right)\right]+2\right) \\
& \geqslant 2\left(\rho\left(\gamma_{n(\beta)}, \gamma_{n(\alpha)}\right)+1\right) \geqslant 2 \rho(\beta, \alpha),
\end{aligned}
$$

hence $\rho(\beta, \alpha) \leqslant k$ and hence

$$
\left\{\bigcup_{|a(\alpha)-n|<k} U_{\alpha} ; n \in \omega\right\} \prec\left\{\bigcup_{\rho(\alpha, \beta)<k} U_{\beta} ; \alpha \in A\right\}
$$

for all $k \in \omega$.

2.3. REMARKs. (a) If $Y$ is a subspace of $X$ and $\mathscr{U}$ is a uniform cover of $Y$, then $\mathscr{U}$ is perfectly refinable in $X$ if and only if the cover $\{U \cup(X \backslash Y) ; U \in \mathcal{Q}\}$ is perfectly refinable.

(b) If $\mathscr{U} \prec \mathcal{V}$ are uniform covers of $Y$ and $\mathscr{U}$ is perfectly refinable in $X$, then $\mathscr{V}$ is perfectly refinable in $X$ as well. On the other hand the meet of two perfectly refinable covers need not be perfectly refinable (see the following example), so the family of all perfectly refinable uniform covers of a space does not form a uniformity in general. Also one can easily observe (see Remark 2.6) that a perfectly refinable uniform cover of a space need not possess a uniform perfectly refinable star-refinement.

2.4. Example. Take $X=\langle 0,1\rangle \times \omega$. The covers $\{\langle 0,1) \times \omega,(0,1\rangle \times \omega\}$, $\{\langle 0,1\rangle \times\{n\} ; n \in \omega\}$ are both obviously perfectly refinable. To see that their meet is not perfectly refinable put

$$
a(\langle 0,1) \times\{n\})=0, \quad a((0,1\rangle \times\{n\})=n,
$$

and observe that for each uniform cover $\mathfrak{V}$ of $X$ there is $k \in \omega$ such that $\mathrm{St}^{k} \mathfrak{V}>\{\langle 0,1\rangle \times\{n\} ; n \in \omega\}$.

The following lemma describes the main connection of perfectly refinable covers with our "in-between" problem.

2.5. LEMMA. Let $f \geqslant g$ be $\bar{R}$-valued functions on a uniform space $X$ such that $f>-\infty, g<+\infty$. Suppose that for each basic uniform cover $\mathcal{Q}$ of $R$ there is $a$ perfectly refinable point-finite uniform cover $\mathfrak{V}$ of $X$ such that $(f, g)^{-1}(\mathscr{U}) \succ \mathfrak{V}$. Then there is a uniformly continuous function $\varphi$ on $X$ such that $f \geqslant \varphi \geqslant g$. 
Proof. (a) The cover $(f, g)^{-1}(\mathcal{T}(\delta / 2))$ can be refined by a uniform perfectly refinable cover $\mathfrak{V}$. For $V \in \mathcal{V}$ take an integer $a(V)$ such that $f(x) \geqslant a(V) \delta-\delta$, $g(x) \leqslant a(V) \delta+\delta$ for all $x \in V$. Since $\rho\left(V_{1}, V_{2}\right)=\left|a\left(V_{1}\right)-a\left(V_{2}\right)\right|$ is a separable pseudometric on $\mathfrak{V}$, there is a uniform cover $\mathscr{W}$ of $X$ refining $\mathfrak{V}$ such that

$$
\mathrm{St}^{k} \text { } \prec \prec\left\{\bigcup_{|a(V)-n|<k} V ; n \text { integer }\right\}
$$

for all $k \in \omega$. Take arbitrary $k \in \omega, k \geqslant 1$ and an integer $n$. Then, for each $x \in \cup_{|a(V)-n| \leqslant k} V$, we have

$$
\begin{aligned}
& f(x) \geqslant n \delta-(k+1) \delta \geqslant n \delta-2 k \delta, \\
& g(x) \leqslant n \delta+(k+1) \delta \leqslant n \delta+2 k \delta,
\end{aligned}
$$

hence

$$
\bigcup_{|a(V)-n|<k} V \subset(f, g)^{-1}(\langle n \delta-2 k \delta, n \delta+2 k \delta\rangle) .
$$

This implies that $\mathrm{St}^{k}$ W refines $(f, g)^{-1}\left(\operatorname{St}^{k}(\mathcal{T}(\delta))\right)$ for all $k \in \omega$ (for $k=0$ it holds since $\mathscr{W} \prec \mathcal{V}$ ). Applying 1.2 and 1.3 we obtain an $\bar{R}$-valued uniformly continuous function $\bar{\varphi}$ such that $f \geqslant \bar{\varphi} \geqslant g$.

(b) Since the sets $\{x \in X \mid \bar{\varphi}(x)=+\infty\},\{x \in X \mid \bar{\varphi}(x)=-\infty\},\{x \in X \mid \bar{\varphi}(x) \in$ $R$ \} form a uniform partition of $X$, we need to consider only the cases $f=+\infty$ or $g=-\infty$. Since the latter case can be obtained from the former one by changing signs only, we may suppose $f=+\infty$. Considering, if necessary, the function $\max (g(x), 0)$, we may also assume that $g \geqslant 0$. Let $\mathcal{V}$ be a point-finite uniform cover of $X$ refining $(f, g)^{-1}(\mathcal{T}(1))$. That means for each $V \in \mathcal{V}$ the function $g$ is upper bounded on $V$. Put $\tilde{f}(x)=\sup \left\{g(u) ; u \in U_{x \in V \in \mathcal{V}} V\right\}$. Since $\mathscr{V}$ is pointfinite, $\tilde{f} \geqslant g$ is finite. For each $V \in \mathcal{V}$, we obviously have that

$$
V \subset\left\{x \in X \mid f(x) \geqslant \sup _{u \in V} g(u), g(x) \leqslant \sup _{u \in V} g(u)\right\},
$$

which implies that for each $\delta>0$ the cover $\mathcal{V}$ refines $(\tilde{f}, g)^{-1}(\mathcal{T}(\delta))$. Applying (a) we finish the proof of the lemma.

2.6. Remark. We note that for finite functions $f, g$ the cover $(f, g)^{-1}(U)$ is obviously point-finite for a linear cover $\mathscr{U}$ of $R$, hence in the case of $R$-valued functions it suffices to assume that $(f, g)^{-1}(\mathscr{Q})$ is uniform and perfectly refinable for each basic cover $\mathcal{Q}$ of $R$.

On the other hand we cannot omit the assumption of point-finiteness in the case of infinite functions; for example if $f=+\infty, g=x^{2}$ on the space $R$, then $(f, g)^{-1}(\mathcal{U})$ is uniform and perfectly refinable for each basic uniform cover $\mathscr{U}$ of $R$, but we cannot find any uniformly continuous function above $g$ on $R$.

2.7. Corollary. Let $X$ be a uniform space such that each countable uniform cover of $X$ is perfectly refinable. Suppose $f \geqslant g$ are $\bar{R}$-valued functions such that $f>-\infty$, $g<+\infty$. Then the following conditions are equivalent:

(1) For each basic uniform cover $\mathcal{Q}$ of $R$ the cover $(f, g)^{-1}(\mathcal{Q})$ is a uniform cover of $X$.

(2) There exists a uniformly continuous real-valued function $\varphi$ on $X$ such that $f \geqslant \varphi \geqslant g$. 
This is a direct consequence of Lemma 2.5 , because each countable uniform cover can be refined uniformly by a countable point-finite cover. It will be seen later (see Theorem 2.13), that the condition imposed on $X$ in Corollary 2.7 is far from being necessary for this type of in-between theorem.

The following theorem shows the connection of perfect refinability and extension property for real-valued functions. Following [4] or [5] we shall denote by Ext the class of all uniform spaces $X$ such that each uniformly continuous function on any subspace of $X$ has a uniformly continuous extension over $X$.

2.8. TheOREM. Let $X$ be a uniform space, $Y$ its subspace. The following conditions are equivalent:

(1) Each uniformly continuous $R$-valued function defined on any subspace of $Y$ has a uniformly continuous extension over $X$.

(2) Each countable finite-dimensional uniform cover of $Y$ is perfectly refinable in $X$.

Proof. $(1) \Rightarrow(2)$. Since $Y \in$ Ext, each countable finite-dimensional uniform cover of $Y$ can be uniformly refined by a star-bounded one (see [1]). Therefore it is sufficient to prove the perfect refinability in $X$ for covers of the form

$$
\left\{F^{-1}\left(\prod_{k=1}^{n}\left\langle\sigma_{k}, \sigma_{k}+3\right\rangle\right) ; \sigma=\left(\sigma_{1}, \ldots, \sigma_{n}\right) \in Z^{n}\right\}
$$

where $F: Y \rightarrow R^{n}$ is a uniformly continuous mapping, $Z$ stands for the set of all integers. Put

$$
\begin{aligned}
I_{\sigma} & =\prod_{k=1}^{n}\left\langle\sigma_{k}, \sigma_{k}+3\right\rangle, \quad \text { for } \sigma \in Z^{n}, \\
K_{z}^{i} & =\prod_{k=1}^{n}\left\langle 3 z_{k}+i_{k}, 3 z_{k}+i_{k}+2\right\rangle, \text { for } z \in Z^{n}, i \in\{0,1,2\}^{n} .
\end{aligned}
$$

Then the following statements easily hold:

(a) For each $i \in\{0,1,2\}^{n}$, the family $\left\{K_{z}^{i} ; z \in Z^{n}\right\}$ is uniformly discrete.

(b) If $x, y \in R^{n}$, then we can find $i \in\{0,1,2\}^{n}$ such that $x, y \in \cup_{z \in Z^{n}} K_{z}^{i}$.

(c) If $x \in R^{n}$ then there is $\sigma \in Z^{n}$ such that $K_{z}^{i} \subset I_{\sigma}$ whenever $x \in K_{z}^{i}$.

Take an arbitrary mapping $a: Z^{n} \rightarrow \omega$ and order the points in $Z^{n}$ into a sequence $\left\{z_{p} ; p \in \omega\right\}$. For $i \in\{0,1,2\}^{n}$ put

$$
\begin{gathered}
b_{i}\left(z_{p}\right)=p \sum\left\{a(\sigma) ; \exists q<p \text { with } K_{z_{q}}^{i} \cap I_{\sigma} \neq \varnothing\right\}, \\
h_{i}(x)=b_{i}(z) \text { for } x \in K_{z}^{i} .
\end{gathered}
$$

Now take a uniformly continuous extension $\gamma_{i}$ of the function $h_{i} \circ(F \uparrow$ $\left.F^{-1}\left(\cup_{z \in Z^{n}} K_{z}^{i}\right)\right)$ over $X$. The cover $\mathfrak{V}=\bigwedge_{i} \gamma_{i}^{-1}\left(\mathcal{T}\left(\frac{1}{4}\right)\right)$ is a uniform cover of $X$. Let $V \in \mathrm{St}^{k} \mathfrak{V}, x \in Y \cap V$. According to (c) we may find $\sigma \in Z^{n}$ such that $K_{z}^{i} \subset I_{\sigma}$ whenever $F(x) \in K_{z}^{i}$.

Take $y \in V \cap Y$. Then $\left|\gamma_{i}(x)-\gamma_{i}(y)\right| \leqslant k$ for all $i \in\{0,1,2\}^{n}$. The assertion (b) now implies the existence of $i \in\{0,1,2\}^{n}$ such that $F(x), F(y) \in \cup_{z} \in Z^{n} K_{z}^{i}$, hence we can find $p, q \in \omega$ such that $F(x) \in K_{z_{p}}^{i}, F(y) \in K_{z_{q}}^{i}$. If $p=q$, then 


$$
\begin{aligned}
& K_{z_{p}}^{i}=K_{z_{q}}^{i} \subset I_{\sigma} . \text { If } p \neq q \text {, choose } \tau \in Z^{n} \text { such that } F(y) \in I_{\tau} \text {. Then } \\
& \qquad \begin{aligned}
k & \geqslant\left|\gamma_{i}(x)-\gamma_{i}(y)\right|=\left|h_{i}(F(x))-h_{i}(F(y))\right| \\
& =\left|b_{i}\left(z_{p}\right)-b_{i}\left(z_{q}\right)\right| \geqslant|a(\sigma)|+|a(\tau)| \\
& \geqslant|a(\tau)-a(\sigma)| .
\end{aligned}
\end{aligned}
$$

Therefore we have

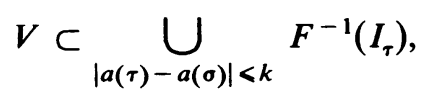

hence the cover $(*)$ is perfectly refinable.

(2) $\Rightarrow(1)$. Take a subspace $B$ of $Y$ and a nonnegative uniformly continuous function $h$ on $B$. There is a countable finite-dimensional uniform cover $\mathfrak{V}$ of $X$ such that $h^{-1}(\mathcal{T}(1 / 2))>\mathrm{St} \mathfrak{V} \uparrow B$. We define two functions $f, g$ on $X$ as follows:

$$
\begin{aligned}
& f(x)=\left\{\begin{array}{l}
1+\sup \{h(y) ; y \in B \cap \operatorname{St}(x, \mathfrak{V})\}, \quad \text { if } B \cap \operatorname{St}(x, \mathfrak{V}) \neq \varnothing, \\
1, \text { otherwise, }
\end{array}\right. \\
& g(x)=\left\{\begin{array}{l}
h(x), \quad \text { for } x \in B, \\
0, \text { otherwise. }
\end{array}\right.
\end{aligned}
$$

$f \geqslant g$ are $R$-valued functions on $X$ and $\mathcal{V}$ refines $(f, g)^{-1}(\mathcal{T}(\delta))$ for all $\delta>0$. Using Lemma 2.5 and Remark 2.3(a) we obtain a uniformly continuous function $\varphi$ on $X$ such that $f \geqslant \varphi \geqslant g$.

We have $h(x) \leqslant \varphi(x) \leqslant h(x)+2$ for $x \in B$, hence the function $h-\varphi$ is bounded on $B$. Let $\psi$ be its uniformly continuous extension over $X$, then $\varphi+\psi$ is uniformly continuous on $X$ and extends $h$. The general case follows from the proved one by decomposing $h$ into the positive and negative parts.

As a consequence of 2.5 and 2.8 we can obtain the following characterization of the class Ext.

2.9. THEOREM. The following properties of a uniform space $X$ are equivalent:

(1) $X \in$ Ext.

(2) Each countable finite-dimensional uniform cover of $X$ is perfectly refinable.

(3) For any two R-valued functions $f \geqslant g$ on $X$ such that for some $\delta>0$ and some countable finite-dimensional uniform cover $\mathfrak{V}$ of $X$ the inequality

$$
\delta+\sup \{g(x) ; x \in V\} \leqslant \inf \{f(x) ; x \in V\}
$$

holds for all $V \in \mathcal{V}$, there is a uniformly continuous function $\varphi$ on $X$ such that $f \geqslant \varphi \geqslant g$.

(4) (In-between theorem for Ext-spaces). For any two $\bar{R}$-valued functions $f \geqslant g$ there is some uniformly continuous function on $X$ in-between $f$ and $g$ if and only if for each basic uniform cover $\mathcal{Q}$ of $R$, the cover $(f, g)^{-1}(\mathcal{U})$ can be refined by a countable finite-dimensional uniform cover of $X$.

Proof. (1) $\Rightarrow$ (2) follows from 2.8 with $X=Y$. (2) $\Rightarrow$ (4) follows immediately from Lemma 2.5. (4) $\Rightarrow(3)$ is obvious; and $(3) \Rightarrow(1)$ can be proved in the same manner as $(2) \Rightarrow(1)$ in Theorem 2.8 . 
2.10. REMARK. If we put $f=+\infty$ in Lemma 2.5 , we obtain directly the following assertion: If each countable monotone uniform cover of $X$ can be uniformly refined by a point-finite perfectly refinable cover, then

For every $\bar{R}$-valued function $g$ on $X$ such that there is a uniform cover $\mathcal{U}$ of $X$ with $g \uparrow U$ upper bounded for all $U \in \mathcal{Q}$, there is an $R$-valued uniformly continuous function $\varphi$ on $X$ such that $\varphi \geqslant g$.

(Under a monotone cover we understand a cover $\left\{U_{n} ; n \in \omega\right\}$ such that $U_{n} \subset$ $U_{n+1}$ for all $n \in \omega$.)

The condition (*) seems to be of some interest, because one can prove the following.

2.11. Proposition. If $X$ enjoys the property (*) from 2.10, then each countable uniform cover of $X$ can be refined by a star-finite uniform cover.

Proof. Take $\mathcal{V}=\left\{V_{n} ; n \in \omega\right\}$, a countable uniform cover of $X$. Let $\mathcal{W}$ be a uniform cover of $X$ refining $\mathcal{V}$ such that each member of $W$ meets only finitely many members of $\mathcal{W}$. Take the function $g(x)=\max \left\{n ; x \in V_{n}\right\} \cdot g$ is bounded on each member of $\Psi$, hence there exists $\varphi$ uniformly continuous such that $\varphi>g$. The cover

$$
\left\{\varphi^{-1}(n-1, n+1) ; n \in \omega\right\} \wedge \mathcal{V}
$$

is a uniform star-finite cover of $X$ and refines $\mathfrak{V}$.

2.12. REMARK. It should be noted that there are spaces in Ext such that not every countable uniform cover has a star-finite uniform refinement. Following [2] we shall call a space $X D^{1}$-locally fine, if each cover of the form $\left\{U_{a} \cap V_{b}^{a}\right\}_{a, b}$ is uniform on $X$, provided that $\left\{U_{a}\right\}_{a}$ is a countable finite-dimensional uniform cover of $X$ and for each $a$ the cover $\left\{V_{b}^{a}\right\}_{b}$ is uniform on $X$. The class of all $D^{1}$-locally fine spaces forms a coreflective subclass of uniform spaces, hence for each uniform space $X$ there is an associated $D^{1}$-locally fine space $\lambda_{D}{ }^{1} X$ which is the coarsest $D^{1}$-locally fine space finer than $X$. For more about these spaces we refer to [14] or [15]. It was a problem of $M$. Rice for some time, whether $\lambda_{D}, M$ is topologically fine (i.e. has the finest uniformity with the same topology as $M$ has) for complete separable metrizable spaces $M$. Recently J. Pelant (unpublished) answered this question negatively. Namely he proved that taking the space $c_{0}$ of null sequences with the usual sup-norm, then $\lambda_{D}{ }^{1} c_{0}$ is not topologically fine. Now it follows from [12], 2.6 and 2.7, that $\lambda_{D}{ }^{1} c_{0}$ is a separable space not having the basis of star-finite covers and of course (see [15]) it is an Ext-space.

The class of all uniform spaces fulfilling the following in-between theorem (2.13, (3) or (4)) is easily strictly smaller than both Ext and (*) from 2.10.

2.13. THEOREM. The following properties of a uniform space $X$ are equivalent:

(1) For each positive $R$-valued uniformly continuous function $\varphi$ on $X$ such that $\inf \{\varphi(x) ; x \in V\}>0$ for each member $V$ of some uniform cover $\mathfrak{V}$ of $X$, the function $1 / \varphi$ is uniformly continuous. 
(2) Each real-valued function $\varphi$ on $X$ such that the covers $\{\{x \in X \mid \varphi(x)<r\}$; $r \in R\}$ and $\{\{x \in X \mid \varphi(x)>r\} ; r \in R\}$ are uniform and if $r, s \in R$ with $r<s$, the sets $\{x \in X \mid \varphi(x)<r\}$, and $\{x \in X \mid \varphi(x)>s\}$ are proximally far in $X$, is uniformly continuous.

(3) If $f \geqslant g$ are $\bar{R}$-valued functions on $X$ such that $f>-\infty, g<+\infty$, the following conditions are equivalent:

(i) The covers $\{\{x \in X \mid g(x)<r\} ; r \in R\}$, and $\{\{x \in X \mid f(x)>r\} ; r \in R\}$ are uniform on $X$ and for each $r, s \in R$ with $r<s$ the sets $\{x \in X \mid f(x)<r\},\{x \in$ $X \mid g(x)>s\}$ are proximally far.

(ii) There exists a uniformly continuous $R$-valued function $\varphi$ on $X$ such that $f \geqslant \varphi \geqslant g$.

(4) If $f \geqslant g$ are $\bar{R}$-valued functions on $X$ such that $f>-\infty, g<+\infty$, the following conditions are equivalent:

(i) $(f, g)^{-1}(\mathcal{Q})$ is uniform for each basic cover थ of $R$.

(ii) There exists a uniformly continuous $R$-valued function such that $f \geqslant \varphi \geqslant g$.

Proof. (1) $\Rightarrow(3)$. Suppose that $f \geqslant g \geqslant 1$. Let $\mathfrak{W}$, $\mathscr{W}$ be uniform covers of $X$ such that $g$ is bounded on each member of $\mathcal{V}$ and each member of $\mathscr{W}$ intersects only finitely many members of $\mathfrak{T}$. Put

$$
f_{1}(x)=\min \{f(x) ; \sup \{g(y) ; y \in \operatorname{St}(x, \mathfrak{V})\}\} .
$$

Then $f \geqslant f_{1} \geqslant g \geqslant 1, f_{1}$ is bounded on each member of $\mho \int$ and moreover the sets $\{x \mid g(x)>a\},\left\{x \mid f_{1}(x)<r\right\}$ are proximally far for all $r<s$. The functions $1 / f_{1}$, $1 / g$ are bounded, $0<1 / f_{1} \leqslant 1 / g \leqslant 1$. If $0<r<s \leqslant 1$, then $1 \leqslant 1 / s<1 / r$, hence the sets

$$
\begin{aligned}
& \left\{x \in X \mid \frac{1}{g}(x)<r\right\}=\left\{x \in X \mid g(x)>\frac{1}{r}\right\} \\
& \left\{x \in X \mid \frac{1}{f_{1}}(x)>s\right\}=\left\{x \in X \mid f_{1}(x)<\frac{1}{s}\right\}
\end{aligned}
$$

are far in $X$. Applying 1.5 we obtain a uniformly continuous function $\psi$ on $X$ such that $1 / f_{1} \leqslant \psi \leqslant 1 / g$; hence for each $W$ in $\mho$, $\inf \{\psi(x) ; x \in W\}>0$. Applying condition (1) we have that $\varphi=1 / \psi$ is uniformly continuous and, of course, $f \geqslant \varphi \geqslant g$. Using translation, we prove the result for $g \geqslant 0$ and, using decompositions into positive and negative parts, we finish the proof of (i) $\Rightarrow$ (ii). The converse (ii) $\Rightarrow$ (i) is evident. The implications $(3) \Rightarrow(2)$ and $(2) \Rightarrow(1)$ are both obvious, (3) $\Leftrightarrow(4)$ is immediate from 1.2 .

We finish this section with the in-between theorem for inversion-closed spaces. A uniform space $X$ is called inversion-closed, if for each positive uniformly continuous $R$-valued function $f, 1 / f$ is also uniformly continuous. This property was studied by many authors; we refer e.g. to [3], where nice characterizations of the class of inversion-closed spaces are proved. Conditions (3), (4), (5) of the following theorem are new interesting properties, characterizing inversion-closedness. One should easily realize again that the class of inversion-closed spaces is much smaller than the class described in Theorem 2.13. 
2.14. THEOREM. The following properties of a uniform space $X$ are equivalent:

(1) $X$ is inversion-closed.

(2) Each real-valued coz-function (i.e. preimages of open sets are in $\operatorname{coz} X$ ) is uniformly continuous.

(3) Each $R$-valued function $\varphi$ such that, if $r<s$ are reals, the sets $\{x \in X \mid \varphi(x) \leqslant$ $r\},\{x \in X \mid \varphi(x) \geqslant s\}$ are proximally far, is uniformly continuous.

(4) (In-between theorem for inversion-closed spaces) If $f \geqslant g$ are $R$-valued functions on $X$, the following conditions are equivalent:

(i) If $r, s \in R$ with $r<s$, the sets $\{x \in X \mid f(x) \leqslant r\},\{x \in X \mid g(x) \geqslant s\}$ are proximally far.

(ii) There is a uniformly continuous function $\varphi$ on $X$ such that $f \geqslant \varphi \geqslant g$.

(5) (In-between theorem for inversion-closed spaces) If $f \geqslant g$ are $\bar{R}$-valued functions on $X$ such that $f>-\infty, g<+\infty$, then the following conditions are equivalent:

(i) If $r, s \in R$ with $r<s$, the sets $\{x \in X \mid f(x) \leqslant r\},\{x \in X \mid g(x) \geqslant s\}$ are $\operatorname{coz} X$-far and the set $\{x \in X \mid f(x)=+\infty\} \cup\{x \in X \mid g(x)=-\infty\}$ can be covered by countably many cozero sets $C_{n}$ such that $g$ is upper bounded and $f$ is lower bounded on each $C_{n}$.

(ii) There exists a uniformly continuous function $\varphi$ on $X$ such that $f \geqslant \varphi \geqslant g$.

Proof. (1) $\Leftrightarrow(2)$ is well known, see [3].

$(2) \Rightarrow(5)$. Suppose (i). First we show that there is a countable cozero cover of $X$ such that $g$ is upper bounded on each of its members. For each $k \in \omega$ choose a cozero set $D_{k}$ such that $D_{k} \supset\{x \in X \mid f(x) \leqslant k\}$ and $D_{k} \cap\{x \in X \mid g(x) \geqslant k+1\}$ $=\varnothing$. Then the family $\left\{C_{n}, D_{n}\right\}_{n, k}$ is the desired cover of $X$. Similarly we find a countable cozero cover of $X$ such that $f$ is lower bounded on each of its members. If we denote by $\tilde{X}$ the uniformity finer than $X$ which arises from $X$ by adding all countable $\operatorname{coz} X$-covers as uniform covers (usually called the (separable metric)fine coreflection of $X$ ), obviously $\tilde{X}$ fulfils condition (1) from 2.13 and $\operatorname{coz} \tilde{X}=$ $\operatorname{coz} X$. Applying Theorem 2.13 (condition (3)) for $\tilde{X}$ we obtain the existence of a cozero function $\varphi$ on $\tilde{X}$ in-between $f$ and $g$ and (2) implies that $\varphi$ is even uniformly continuous on $X$. (ii) $\Rightarrow$ (i) is obvious, as well as the implications (5) $\Rightarrow(4) \Rightarrow(3) \Rightarrow$ (1).

3. Applications. First we present an application to the problem of approximation of a function by a uniformly continuous one. Theorem 3.1 is an improvement of the Hahn's Lemma [6] (for a generalization and applications see [1]); its proof also shows a method of using the in-between theorems in proving results of similar type.

3.1. TheOREM. Let $h$ be a real-valued function on a uniform space $X$ and let $\varepsilon=\inf \left\{\delta>0 ; h^{-1}(\mathcal{T}(\delta))\right.$ is uniform $\}$. Then $\varepsilon$ is the least number for which there exists a uniformly continuous function $\varphi$ with $|h-\varphi| \leqslant \varepsilon / 2$.

Proof. First observe that $|h-\psi| \geqslant \varepsilon / 2$ for each uniformly continuous function $\psi$; hence it is sufficient to show that there is a uniformly continuous function in-between $f=h+\varepsilon / 2$ and $g=h-\varepsilon / 2$. Let us verify condition (3) of 1.4 for the pair $f, g$. 
Take $\delta>0$ and choose a natural number $k$ such that $k>1+\varepsilon / \delta$. We can find a uniform cover $\mathcal{V}$ of $X$ such that $\mathrm{St}^{k} \mathcal{V}$ refines the cover $h^{-1}(\mathcal{T}(\varepsilon+\delta))$. Let $x, y \in V \in \mathrm{St}^{n} \mathfrak{V}$. Then there are $V_{1}, V_{2}, \ldots, V_{2 n} \in \mathfrak{V}$ such that $x \in V_{1}, y \in$ $V_{2 n}$ and $V_{i-1} \cap V_{i} \neq \varnothing$ for $i=2, \ldots, 2 n$. If we take a sequence $\left\{x_{i} ; 1 \leqslant i \leqslant 2 n\right.$ $+1\}$ of points in $X$ such that $x_{1}=x, x_{2 n+1}=y$ and $x_{i} \in V_{i-1} \cap V_{i}$ for $i=$ $2,3, \ldots, 2 n$, we have

$$
\left|h\left(x_{i}\right)-h\left(x_{j}\right)\right|<\varepsilon+\delta
$$

whenever $|i-j| \leqslant 2 k+1$. Choosing a natural number $m$ such that $m k>n \geqslant$ $(m-1) k$, we obtain

$$
\begin{aligned}
g(y)-f(x) & =h(y)-h(x)-\varepsilon \leqslant m(\varepsilon+\delta)-\varepsilon \\
& \leqslant \delta+(n / k)(\varepsilon+\delta)<(n+1) \delta .
\end{aligned}
$$

The following application allows us to construct some Ext-uniformities on the real line. It is proved in [4], [5] that if $R^{\prime}$ is a uniformity on the real line finer than $R$ such that $R^{\prime}$ is a value of $R$ under some coreflector in the category of uniform spaces and $R^{\prime} \in$ Ext, then $R^{\prime}$ is finer than the topologically fine uniformity $t_{f} R$ (having for basis all open covers of $R$ ). However it was a problem, whether (omitting the first assumption) one can find some Ext-uniformity on the real line finer than $R$ and strictly coarser than $t_{f} R$. Theorem 3.2 gives even infinitely many such uniformities.

3.2. TheOREM. Let $D$ be an infinite uniformly discrete subset of $R$. Let $\nu$ be the family of all open covers $\mathcal{Q}$ of $R$ for which there is $\varepsilon>0$ such that for each $d \in D$ one can find $U \in \mathcal{Q}$ with $(d-\varepsilon, d+\varepsilon) \subset U$. Then $\nu$ is a basis of an Ext-uniformity that is finer than $R$ and strictly coarser than $t_{f} R$.

Proof. The family $\nu$ is obviously closed under meets. Moreover, each cover from $\nu$ can be refined by a cover $\mathcal{U}=\left\{\left(a_{n}, b_{n}\right) ; n \in Z\right\}$ such that $a_{n}<b_{n-1}<a_{n+1}$ for all $n \in Z$ and, for some $\delta>0$ and each $d \in D$ there is a unique integer $n$ with $b_{n-1} \leqslant d-\delta<d+\delta \leqslant a_{n+1}$. Let $\kappa: Z \rightarrow \omega$ be an arbitrary mapping. Take a subset $S$ of $\cup_{n=-\infty}^{+\infty}\left(a_{n}, b_{n-1}\right)$ such that

$$
\operatorname{card}\left(S \cap\left(a_{n}, b_{n-1}\right)\right)=2(1+|\kappa(n)-\kappa(n-1)|) .
$$

Let $\mathcal{V}$ be the cover of $R$ consisting of those open intervals with end-points in $S$ which contain exactly one point of $S$. Then $\mathfrak{V} \in \nu$ and

$$
\mathrm{St}^{k} \mathcal{V} \prec\left\{\bigcup_{|\kappa(n)-p|<k}\left(a_{n}, b_{n}\right) ; p \in \omega\right\}
$$

for all $k \in \omega$. This implies that $\nu$ is a basis of some uniformity on the real line which is in Ext because of Theorem 2.9 and Proposition 2.2. The remaining assertions are obvious.

3.3. REMARK. Observe that for each open cover $\mathfrak{V}$ of $R$, which is not (metrically) uniform, we can find (by induction) an infinite uniformly discrete subset of $R$ with the property that for any $\varepsilon>0$, there is $d \in D$ such that no $V \in \mathcal{V}$ contains the interval $(d-\varepsilon, d+\varepsilon)$. The preceding theorem constructs an Ext-uniformity finer than $R$, coarser than $t_{f} R$ and such that $\mathscr{T}$ is not uniform. 
From Theorem 2.14 we can easily deduce the following in-between theorem in topological spaces.

3.4. THEOREM. Let $f \geqslant g$ be $\bar{R}$-valued functions on a topological space $X$ and let $f>-\infty, g<+\infty$. Then the following conditions are equivalent:

(1) If $r, s \in R$ with $r<s$, then the sets $\{x \in X \mid f(x)<r\},\{x \in X \mid g(x) \geqslant s\}$ can be separated by zero sets and there is a countable cozero cover of the set $\{x \in X \mid f(x)$ $=+\infty\} \cup\{x \in X \mid g(x)=-\infty\}$ such that $g$ is upper bounded and $f$ is lower bounded on each of its members.

(2) There exists a continuous function $\varphi$ on $X$ such that $f \geqslant \varphi \geqslant g$.

Proof. Take the Hausdorff modification of the uniformity projectively generated by all continuous functions and use $2.14(5)$.

As corollaries of the general Theorem 3.4 we may obtain the following three known in-between theorems for special topological spaces.

3.5. Corollary [8]. Let $X$ be a Hausdorff topological space. The following conditions are equivalent:

(1) $X$ is normal.

(2) If $f \geqslant g$ are $R$-valued functions on $X$ such that $f$ is lower semicontinuous and $g$ is upper semicontinuous, then there exists a continuous function $\varphi$ on $X$ with $f>\varphi>$ g.

Proof. (1) $\Rightarrow$ (2) follows from 3.4 since for $r<s$ the sets $\{x \in X \mid f(x)<r\}$, $\{x \in X \mid g(x) \geqslant s\}$ are disjoint and closed, hence they can be separated by zero sets. The converse is evident.

3.6. COROLlary [8]. The following properties of a Hausdorff topological space $X$ are equivalent:

(1) $X$ is countably paracompact normal.

(2) If $f \geqslant g$ are $\bar{R}$-valued functions on $X$ such that $f>-\infty, g<+\infty$, $f$ is lower semicontinuous and $g$ is upper semicontinuous, then there exists a continuous function $\varphi$ on $X$ such that $f \geqslant \varphi \geqslant g$.

(3) If $f>g$ are $\bar{R}$-valued functions on $X$ such that $f$ is lower semicontinuous and $g$ is upper semicontinuous, then there exists a continuous function $\varphi$ on $X$ such that $f>\varphi>g$.

Proof. $(1) \Rightarrow(2)$. Since $X$ is normal, the first part of condition (1) in 3.4 is obvious. Moreover the countable paracompactness implies that the open covers $\{\{x \in X \mid f(x)>n\} ; n \in \omega\}$ and $\{\{x \in X \mid g(x)<-n\} ; n \in \omega\}$ of $X$ can be refined by cozero covers, which implies the second part of 3.4(1).

(1), (2) $\Rightarrow(3)$. Let $\tilde{X}$ be the corresponding topologically-fine uniform space of $X$. In view of the countable paracompactness of $X$ the cover $\{\{x \in X \mid f(x)>r>s>$ $g(x)\} ; r<s$ rational numbers $\}$ can be refined by a countable cozero cover. Now we can apply 1.7. Conditions (2) or (3) immediately imply that each monotone countable open cover of $X$ can be refined by a countable cozero cover of $X$; this implies the countable paracompactness of $X$ (for details see [8]). 
Note that there are examples of normal spaces not being countably paracompact, see e.g. [13].

3.7. Corollary [10]. Let $X$ be a Hausdorff topological space, the following conditions are equivalent:

(1) $X$ is perfectly normal.

(2) If $f \geqslant g$ are $\bar{R}$-valued functions on $X$ such that $f$ is lower semicontinuous and $g$ is upper semicontinuous, then there is a continuous function $\varphi$ on $X$ such that $f \geqslant \varphi \geqslant g$ and $f(x)>\varphi(x)>g(x)$ whenever $f(x)>g(x)$.

Proof. Observe that perfect normality implies countable paracompactness, hence 3.6 together with 1.7 prove $(1) \Rightarrow(2)$. The converse is easy.

Topological methods have been used in the theory of real functions of one real variable for quite a long time and have led to nice results concerning the construction of derivatives (see e.g. [11], [16]). Their proofs use only topological ideas and not theorems however, since the density topology is not normal. Here we intend to show that the use of uniformities may overcome this difficulty; it will easily give us a generalization of Theorem 7 from [16] and thus a solution of Problem III from [16], as well as a generalization of Theorems 3.2 and 4.14 from [11] and a solution of Problem 5.1.3 from [11]. In what follows we shall call the Lebesgue measure on $R$, the Lebesgue integral, etc., simply the measure, integral, etc. Recall that a set $E \subset R$ is an $M_{5}$-set if it is an $F_{0}$-set consisting only of its points of density (i.e. $\lim _{h \rightarrow 0}(1 / h)|E \cap(x, x+h)|=1$ for each $x \in E$, where $|M|$ denotes the measure of $M$ ). A map $\varphi: R \rightarrow M$, where $M$ is a separable metric space, is called approximately continuous if $\varphi^{-1}(G) \in M_{5}$ for each open set $G \subset M$. (For other definitions see e.g. [16].)

The main tool in working with these notions is the following lemma due to Luzin and Menchoff (see e.g. [9]).

3.8. LeMMA. Let $E \subset R$ be a measurable set, $c \in R, c>0$. Suppose that $F \subset E$ is a closed set. Then there is a closed set $H \subset E$ such that $F \subset H$ and $\mid(x, x+h) \cap$ $(E-H) \mid \leqslant c h^{2}$ whenever $x \in F$ and $h \neq 0$.

Proposition. The family of all countable covers of $R$ with $M_{5}$-sets is a basis of a uniformity.

Proof. We need to show that every cover $\mathscr{U}=\left\{E_{n} ; n \in \omega\right\}$ of $R$, such that $E_{n} \in M_{5}$ for each $n \in \omega$, can be star refined by a countable $M_{5}$-cover. Assume first that $\mathscr{Q}$ is star-finite. From the preceding lemma it follows that there are closed sets $F_{n, k}$ and $M_{5}$-sets $H_{n, k}$ such that $\varnothing=F_{n, 0} \subset H_{n, 0} \subset F_{n, 1} \subset H_{n, 1} \subset \cdots$ and $E_{n}=$ $\cup_{k=0}^{\infty} F_{n, k}$. Consider the cover of $R$ by sets of the form $\left(\cap_{n \in A}\left(H_{n, k_{n}+1}-F_{n, k_{n}}\right)\right)$ - $\cup_{n \notin A} F_{n, m+2}$, where $A$ runs through all nonempty finite subsets of $\omega,\left\{k_{n}\right\}$ runs through all sequences of natural numbers and $m=\max \left\{k_{n} ; n \in A\right\}$. Since $\mathcal{Q}$ is star-finite, these sets belong to $M_{5}$ and form a cover $\mathcal{V}$ of $R$. For $x \in R$ choose the least $k \in \omega$ such that $x \in H_{p, k}$ for some $p \in \omega$. If $x \in\left(\bigcap_{n \in A}\left(H_{n, k_{n}+1}-F_{n, k_{n}}\right)\right)-$ $\cup_{n \notin A} F_{n, m+2}$ then $k \leqslant k_{n}+1$ for each $n \in A$, hence $k<m+1$. Since $H_{p, k} \subset$ $F_{p, m+2}$ this implies $p \in A$. Thus $\operatorname{St}(x, \mathcal{V}) \subset E_{p}$. If $U$ is not star-finite, we can use its 
star-finite refinement $\left\{H_{n, k+1}-\cup_{p+q<n+k} F_{p, q} ; n, k \in \omega\right\}$.

The uniformity from the preceding proposition will be called the density uniformity. Obviously a mapping from $R$ with the density uniformity into a separable metric space is uniformly continuous if and only if it is approximately continuous. It follows that the density uniformity is inversion closed, hence we may construct some approximately continuous functions with the help of Theorem 2.14.

3.9. Proposition. $A$ set $E \subset R$ is a cozero set in the density uniformity if and only if $E \in M_{5}$.

Proof. If $E \in M_{5}$ let $\left\{F_{n}\right\}$ be a sequence of closed sets such that $F_{n} \subset F_{n+1}$ and $E=\cup_{n} F_{n}$. Put $g(x)=1 /(n+1)$ for $x \in F_{n}-F_{n-1}, f(x)=1$ for $x \in E$ and $g(x)=f(x)=0$ for $x \notin E$. For $0 \leqslant r<s \leqslant 1$ the sets $\{x \in R ; f(x) \leqslant r\},\{x \in$ $R ; g(x) \geqslant s\}$ are $\mathcal{W}$-far for $\mathcal{V}=\left\{E, R-F_{n}\right\}$ with $1 / n<s$. According to Theorem 2.14 there is a uniformly continuous function $\varphi$ such that $f \geqslant \varphi \geqslant g$. The converse statement is obvious.

One of the main results of [16] is a special case of the following theorem, for $f, g$ acquiring only finitely many values. Hence 3.10 solves Problem III from [16] (to generalize this result for functions acquiring more than finitely many values). For convenience put $a^{+}=\max \{a, 0\}, a^{-}=\max \{-a, 0\}$ for $a \in \bar{R}$.

3.10. THEOREM. Let $F$ be a continuous increasing function on $\langle 0,+\infty)$ such that $F(0)=0$. Let $f \geqslant g$ be $\bar{R}$-valued functions on $R$ such that the sets $\{x \in R$; $f(x)>r\}, \quad\{x \in R ; g(x)<r\}$ belong to $M_{5}$ for each $r \in R$. Suppose that $\lim _{h \rightarrow 0}(1 / h) \int_{x}^{x+h}\left(F\left((g(t)-a)^{+}\right)\right) d t=0$ whenever $g(x)<a<+\infty$ and $\lim _{h \rightarrow 0}(1 / h) \int_{x}^{x+h} F\left((f(t)-a)^{-}\right) d t=0$ whenever $f(x)>a>-\infty$. Then there exists an $\bar{R}$-valued measurable function $\varphi$ on $R$ such that

(1) $f \geqslant \varphi \geqslant g$ and $f(x)>\varphi(x)>g(x)$ whenever $f(x)>g(x)$,

(2) $\lim _{h \rightarrow 0}(1 / h) \int_{x}^{x+h} F\left((\varphi(t)-c)^{+}\right) d t=0$ for each $c>\varphi(x), c \in R$, and

(3) $\lim _{h \rightarrow 0}(1 / h) \int_{x}^{x+h} F\left((\varphi(t)-c)^{-}\right) d t=0$ for each $c<\varphi(x), c \in R$.

Proof. Let $\left\{a_{n} ; n \in \omega\right\}$ be an increasing sequence of positive real numbers such that $\lim _{n \rightarrow+\infty} a_{n}=+\infty$ and $F\left(a_{k+1}-c\right) \leqslant 2 F\left(a_{k}-c\right)$ for each $k \in \omega$ and $c \in R$ with $2|c| \leqslant a_{k}$. (Its existence follows from the fact that the sequence defined by $a_{0}=1, a_{k+1}=\sup \left\{a ; F(a-c) \leqslant 2 F\left(a_{k}-c\right)\right.$ for $\left.2|c| \leqslant a_{k}\right\}$ cannot be bounded.)

For each $n \in \omega$ choose closed sets $F_{n, k}$ such that $\left\{x \in R ; g(x)<a_{n}\right\}=$ $\cup_{k} F_{n, k}$. Let $H_{m} \supset \cup_{n, k \leqslant m} F_{n, k}$ be closed sets such that $H_{m} \subset\{x \in R$; $\left.g(x)<a_{m}\right\}$ and

$$
\left|\left\{t \in(x, x+h) ; g(t)<a_{m}\right\}-H_{m}\right|<\left(F\left(m+1+a_{m+1}\right)\right)^{-1} 2^{-m} h^{2}
$$

for each $x \in \cup_{n, k<m} F_{n, k}$ and $h \neq 0$.

Put $\tilde{f}(x)=\inf \left\{a_{m} ; x \in H_{m}\right\}$ (hence $f(x)=+\infty$ if $\left.g(x)=+\infty\right)$. Similarly we construct the function $\tilde{g}$. Put $f_{1}=\min \{f, \tilde{f}\}, g_{1}=\max \{g, \tilde{g}\}$. Then $f_{1}>g_{1}, f_{1}(x)$ $>g_{1}(x)$ whenever $f(x)>g(x)$ and the sets $\left\{x \in R ; f_{1}(x)>r\right\},\left\{x \in R ; g_{1}(x)<r\right\}$ belong to $M_{5}$ for each $r \in R$. Theorems 1.5 and 1.7 imply the existence of an approximately continuous function $\psi$ on $R$ such that $\operatorname{arctg} g_{1}<\psi \leqslant \operatorname{arctg} f_{1}$ and $\operatorname{arctg} g_{1}(x)<\psi(x)<\operatorname{arctg} f_{1}(x)$ whenever $g(x)<f(x)$. Put $\varphi=\operatorname{tg} \psi$. Then (1) is 
clear, let us prove (2). If $c>\varphi(x), c \in R$ and $m \in \omega$ such that $x \in \cup_{n, k<m} F_{n, k}$ and $2|c|<m$ then

$$
\begin{aligned}
& \int_{(x, x+h)} F\left((\varphi(t)-c)^{+}\right) d t=\int_{(x, x+h) \cap H_{m}} F\left((\varphi(t)-c)^{+}\right) d t \\
& \quad+\sum_{k=m+1}^{\infty} \int_{(x, x+h) \cap\left(H_{k}-H_{k-1}\right)} F\left((\varphi(t)-c)^{+}\right) d t \\
& \leqslant F\left(\left(a_{m}-c\right)^{+}\right)|\{t \in(x, x+h) ; \varphi(t)>c\}| \\
&+\sum_{k=m+1}^{\infty} F\left(\left(a_{k}-c\right)^{+}\right)\left|\left\{t \in(x, x+h) ; a_{k-1} \leqslant g(t)<a_{k}\right\}\right| \\
&+\sum_{k=m+1}^{\infty} F\left(\left(a_{k}-c\right)^{+}\right)\left(F\left(k+a_{k}\right)\right)^{-1} 2^{-k} h^{2} \\
& \leqslant F\left(\left(a_{m}-c\right)^{+}\right)|\{t \in(x, x+h) ; \varphi(t)>c\}| \\
&+2 \int_{(x, x+h)} F\left((g(t)-c)^{+}\right) d t+h^{2} .
\end{aligned}
$$

Now (2) follows from the approximate continuity of $\psi$ and the assumptions of the theorem. The proof of (3) is similar.

A more detailed discussion of applications of uniformities in the theory of real functions will be contained in a separate paper. Let us just mention that the function from the preceding theorem is obviously approximately continuous (as a mapping into $\bar{R}$ with a metric that makes $\bar{R}$ the usual two-point compactification of $R$ ) and it is the derivative of its indefinite integral provided that $\lim \inf _{x \rightarrow+\infty} F(x) / x>0$. Hence we obtain, as a special case, the following generalization of Theorems 3.2 and 4.14 from [11].

3.11. Corollary. Let $h$ be a function of the first class defined on $R$, and let $E \subset R$ be of measure zero. Then there is a locally integrable approximately continuous function $\varphi$ on $R$ such that $\varphi$ is the derivative of its indefinite integral and $\varphi(x)=h(x)$ for each $x \in E$.

Proof. Let $G \supset E$ be a $G_{\delta}$-set of measure zero. Put $f(x)=+\infty, g(x)=-\infty$ for $x \notin G$ and $f(x)=g(x)=h(x)$ for $x \in G$. Now use the preceding theorem with $F(t)=t$.

Note that 3.11 is precisely the answer to the Problem 5.1.3 from [11].

\section{REFERENCES}

1. H. H. Corson and J. R. Isbell, Some properties of strong uniformities, Quart. J. Math. Oxford Ser. 11 (1960), 17-33.

2. Z. Frolik, Locally e-fine measurable spaces, Trans. Amer. Math. Soc. 196 (1974), 237-247.

3. __ Three uniformities associated with uniformly continuous functions, Sympos. Math., vol. 17, Academic Press, London and New York, 1976, pp. 69-80.

4. Z. Frolík, J. Pelant and J. Vilimovský, On hedgehog-topologically fine uniform spaces, Seminar Uniform Spaces (Prague, 1975-76), Mat. Ústav Ceskaslovenské Akad. Věd., Prague, 1976, pp. 75-86.

5. Extensions of uniformly continuous functions, Bull. Acad. Polon. Sci. Sér. Sci. Math. Astronom. Phys. 26 (1978), 143-148.

6. H. Hahn, Über halbstätige und unstetige Funktionen, Sitzungsb. Akad. Wien 126 (1917), 91-110. 
7. J. R. Isbell, Uniform spaces, Math. Surveys, no. 12, Amer. Math. Soc., Providence, R. I., 1964.

8. M. Katĕtov, On real-valued functions in topological spaces, Fund. Math. 38 (1951), 85-91.

9. I. Maximoff, On density points and approximately continuous functions, Tôhoku Math. J. 47 (1940), 237-250.

10. E. Michael, Continuous selections. I, Ann. of Math. (2) 63 (1956), 361-382.

11. G. Petruska and M. Laczkovich, Baire 1 functions, approximately continuous functions and derivatives, Acta Math. Acad. Sci. Hungar. 24 (1973), 383-387.

12. M. D. Rice, Composition properties in uniform spaces, Acta Math. Acad. Sci. Hungar. 30 (1977), 189-195.

13. M. E. Rudin, $A$ normal space $X$ for which $X \times I$ is not normal, Fund. Math. 73 (1971), 179-186.

14. J. Vilimovský, Uniformly continuous Banach-valued mappings, Proc. Fourth Prague Topological Sympos. (1976), Soc. Czech. Math. and Phys., Prague, 1977, pp. 481-484.

15. , Modules of uniformly continuous Banach-valued mappings, Acta Math. Acad. Sci. Hungar. (to appear).

16. Z. Zahorski, Sur la première derivée, Trans. Amer. Math. Soc. 69 (1950), 1-54.

Faculty of Mathematics and Physics, Charles University, Sokolovskí 83, 18600 Prague 8, Czechoslovakia

Institute of Mathematics, Czechoslovak ACademy of Sciences, Żrtná 25, 11567 Prague 1, Czechoslovakia 